\title{
PilsenCUBE-II Measurement Board as interface for student's experiments
}

\author{
Luděk Dudáček $^{1}$, Aleš Voborník ${ }^{2}$, Jiří Masopust ${ }^{3}$ \\ Faculty of Electrical Engieneering \\ University of West Bohemia \\ Pilsen, Czech Republic \\ 1'dudacekl@kae.zcu.cz, ${ }^{2}$ vobornik@ket.zcu.cz, ${ }^{3}$ masopust@kae.zcu.cz
}

\begin{abstract}
One of the main goals of the PilsenCUBE-II picosatellite project is to offer on board payload space for the student's experiments for the students from the various secondary schools of the western Bohemia. The students experiments have to be simple enough in scope of skills and abilities of the secondary schools students but should provide interesting results for satellite project. There is 6 student's experiments in the second round of competition. This experiments will be integrated into satellite as a parts of the measurement board (MES). The measurement board will provide the interface between these student's experiments (STE) and On-Board Computer (OBC) that is controlling the functions of the satellite and providing the communication with ground station.
\end{abstract}

\section{Keywords-PilsenCUBE-II; Picosatellite}

\section{INTRODUCTION}

In the spring of the 2017 the picosatellite project PilsenCUBE-II was started at the Department of Applied Electronics and Telecommunications in cooperation with Information Technologies Management of the Pilsen City (SITMP).

PilsenCUBE-II is the satellite of the Cube family in $1 \mathrm{U}$ configuration. The overall dimensions of this PilsenCUBE-II satellite are $10 \times 10 \times 10$ centimeters with weight about $1.33 \mathrm{~kg}$. The main parts of the satellite are:

- Power System (PWR),

- On-Board Computer (OBC) combined with standard communication radio (COM1),

- Measurement Board (MES),

- $2.4 \mathrm{GHz}$ software defined radio (SDR),

- Medipix (MED)

This paper is focused on Measurement Board. Measurement board is composed from a number of parts. The Fig.1 shows the parts of the MES. For better overlook there are hidden some parts of the satellite that have not relation with MES, but spatial layout is respecting all satellite systems and parts. Red color is highlighting main MES board, boards for microcontroller tests are in blue, solar modules are highlighted by yellow. Black is main satellite bus and its connector. Camera $\mu C A M$ is purple and the mechanical parts of the satellite are gray.

As was mentioned in the abstract, one of the main aims of the PilsenCUBE-II project is to provide payload place for in orbit experiments performed by the

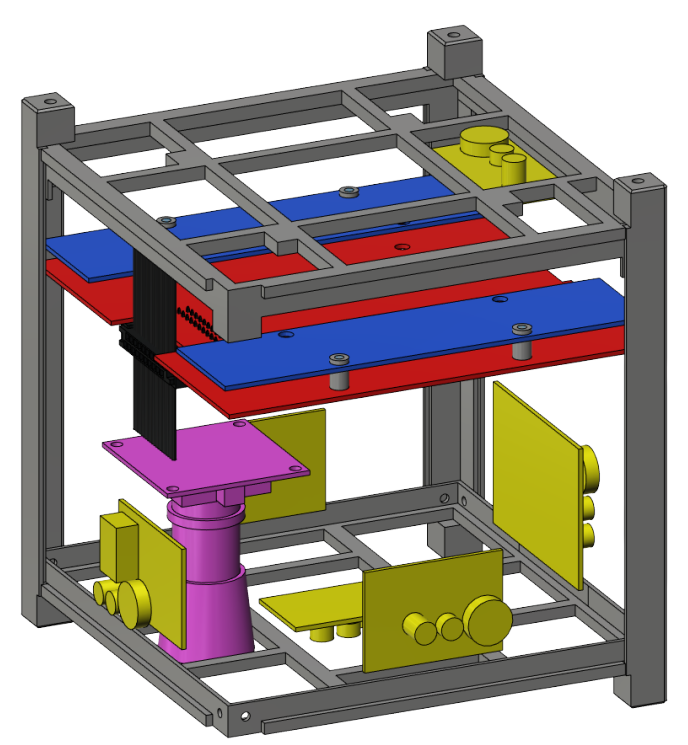

Fig. 1. MES board parts of the satellite.

students from the secondary schools of the western Bohemia. It was organized in the form of competition. From the first round, where students present their ideas, 6 teams were accepted into the second round. In the second round students are preparing prototypes of their experiments. Sufficient hardware is chosen and it's embedding into satellite is prepared as a part of the satellite measurement board. In this section the 6 students experiments (STE) are briefly described:

\section{A. Teploměrka}

Aim of this project prepared by students of the Grammar School of Domazlice is an experimental use of infra-red (IR) sensors (Melexis MLX90621 or Panasonic AMG88) for sensing of IR radiation of the Earth and Sun and experimental evaluation of the satellite attitude and horizon detection from this measurement.

\section{B. SPUT_N1K}

This part of the PilsenCUBE project is developed by students the second team from Grammar School of Domazlice and it's goal is tree axis measurement of the satellite rotation and consequent realization of the virtual model for visualization of the satellite rotation. 
There will be used ICM-20602 Motion Tracker including 3-axis gyroscope and 3-axis accelerometer.

\section{C. iSOUEsense}

iSOUEsense is an experiment performed by students from Secondary Vocational School of Electrical Engineering in Pilsen. This experiment is focused on behaviour of the four various microprocessors (ATMEGA328, STM32L151, ATSAML21, STM32F030) in the space environments. There will be realized functionality checks and recovery after radiation event.

\section{Filip Novotný team}

Secondary school student Filip Novotný is preparing simple semiconductor geiger-muller counter for the measurement of the radiation inside the satellite body. The TEVISO Radiation Sensor BG51 will be used for this purpose as a part of the measurement board.

\section{E. Naděnky team}

Student team from the Secondary School of Electrical Engineering in Pilsen is working on the magnetometer measurement using MMC5883MA device. Measurement results from this sensor will be used for simple stabilization using single magnetic coil.

\section{F. PilsCam}

CMOS camera $\mu$ CAM-III will be installed in the PilsenCUBE-II satellite for team of the students from Secondary School of Informatics and Financial Services in Pilsen, who will take a photo of the Earth. Main aims of this experiment is to test filters protecting the camera against thermal damage and preform the image processing algorithms validating content of the picture and making decision whether the picture should be downloaded to the Earth or not.

\section{G. Non-Students experiments}

There are several another experiments, that are not performed by secondary school students. This experiments will be called NSE (Non-Students Experiment) in scope of this paper. They are prepared by various teams, but their embedding into satellite will be realized through MES board as well as STEs embedding. So in scope of the satellite these experiments will be part of the MES board. These experiments are:

- voltage reference sources testing in space environment,

- in orbit tests of the FRAM based MSP430 microcontrollers,

- radiation measurement performed by Rigaku company,

- in orbit tests of the chemical sensors.

\section{MEASUREMENT BOARD}

Measurement board is one of the payloads connected to satellite backbone bus based on RS485. Main functions of MES board are:

- to switch on and off connected experiments according to commands from OBC scheduler,

- to controll of the running STEs or NSEs,
- to collect and storing data from connected STEs and NSEs,

- to collect and storing data from Solar Modules (SM),

- to preprocess of the data from connected experiments,

- to provid data from connected experiments to OBC.

\section{A. Electrical design}

Because there is large number of connected secondary experiments from various teams, it is necessary to found the design with sufficient immunity for connected experiment failure. We can expect that some of the connected experiments will crash soon in space environment. Failure of the one experiment should not influence function of other experiments. The basic concept of MES board is depicted in the Fig.2 The MES controller is based on STM32F103 micro-

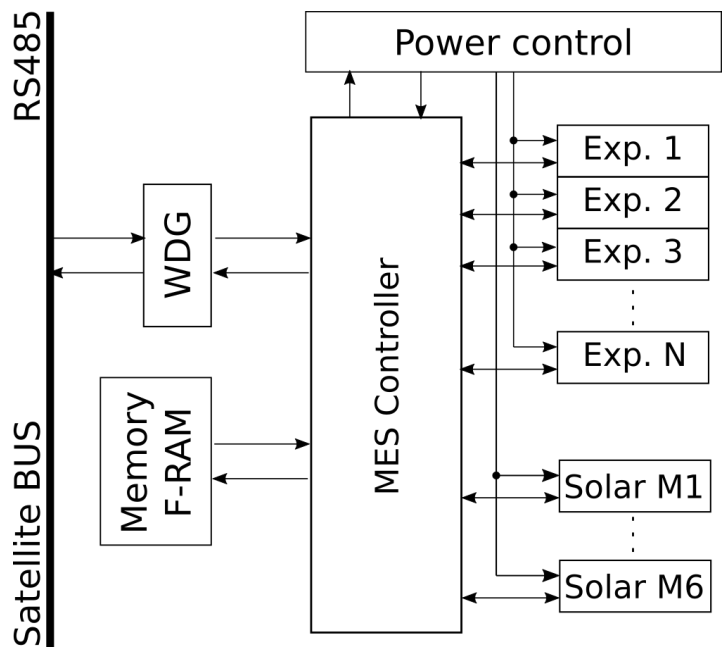

Fig. 2. Basic concept of the MES board.

controller which is connected to satellite bus through watchdog. The watchdog is securing communication on the satellite bus against blocking due to payload failure. It is necessary to update WDG periodically by the MES controller during transmitting. If WDG is not updated correctly, connection to the satellite bus will be switched to the receiver only mode. In more detail is the WDG circuit shown in the figure 3.

Every experiment is connected to the MES board via two channels: Information channel and Power channel.

\section{B. Information channels}

Information channel is providing data connection of the experiment with MES control. There is number of various information channels depending on the requirements of the experiment and requirements of the MES controller. The information channels are realized through standard $I^{2} C$, SPI, USART or GPIO interfaces of the STM32F103. The experiments are connected to the MES controller trough switches that enables disconnecting of the experiment or devices from the information channel. The Fig.4 shows the block scheme of the information channel using $I^{2} C_{1}$. 

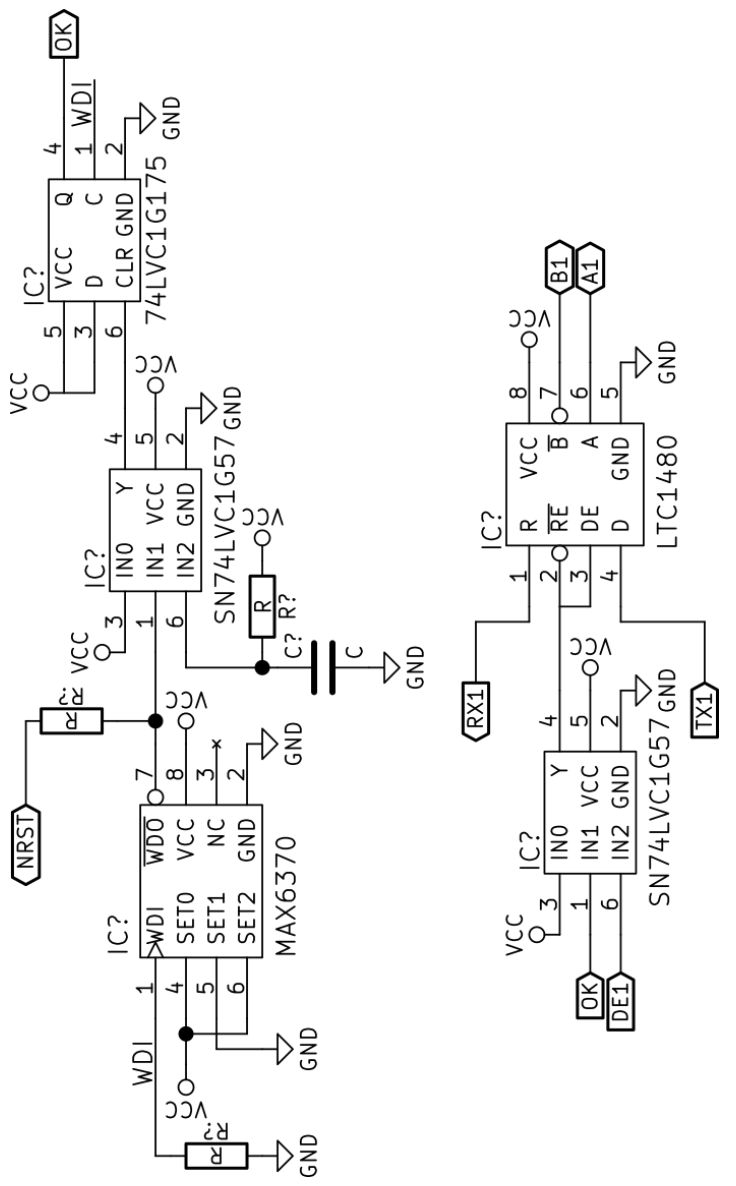

Fig. 3. Watchdog circuit.

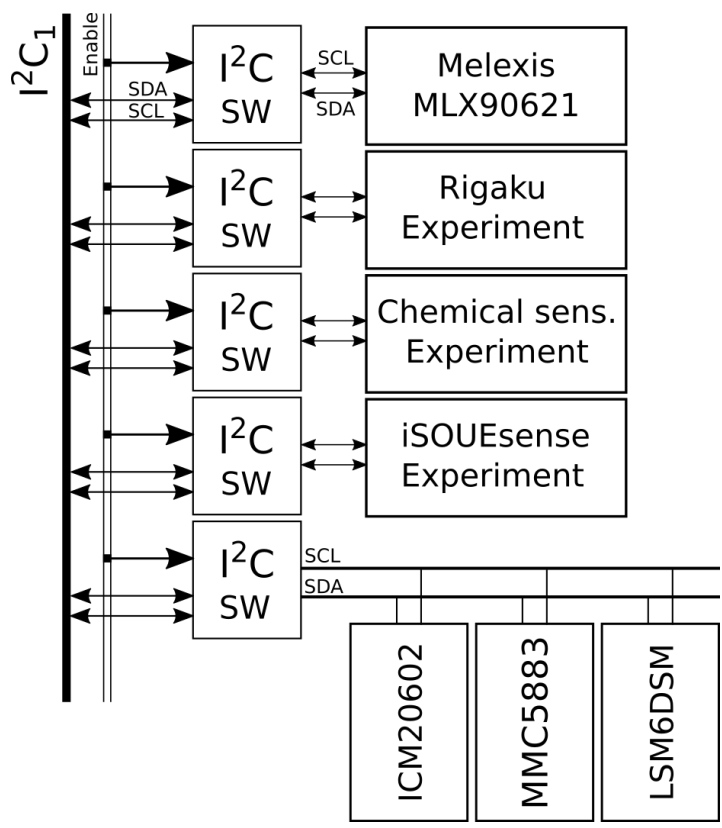

Fig. 4. Information channel using $I^{2} C_{1}$

Information channel $I^{2} C_{1}$ is used for connecton of the solar modules SM1 to SM6. Experiment PilsCam is connected via USART2, tested MSP430 and experiment testing voltage references are connected via $S P I_{1}$ with switches for selective disconnecting of each experiment.
The typical $I^{2} C_{1}$ connection of the experiment to the MES Controller is shown in the Fig.5. The $I^{2} C_{1}$ switches are based on the integrated circuit PCA9517. The left side of this IC is connected to MES Controller $I^{2} C_{1}$ and is powered by VEXP voltage. The right side is an experiment side and usually is powered by the EXPP voltage. The VEXP and the EXPP are described in more detail in the following section of this paper.

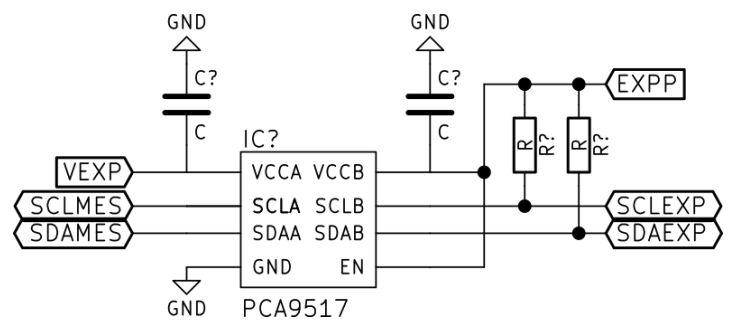

Fig. 5. $I^{2} C_{1}$ Information channel switch.

\section{Power channels}

Power consumption of the satellite payloads is crucial parameter that should be considered carefully. There are three independent unstabilized power buses in the satellite (PWR1 to PWR3) accessible in all payloads. The MES board is connected to all of these PWR buses and is coupling all of them to single power channel called in scope of this paper MES Main Power bus (MES-MP bus).

Power supply of the MES controller, Solar Modules and experiments is realized by power channels derived from this MES-MP bus. Because the failure of any experiment can cause abnormal power consumption, there is power switch in every power channel. This switch is limiting maximal current and can be switched of by MES controller if experiment has to be switched off to prevent any power consumption.

Power system of the MES board is schematically illustrated in the Fig. 6 There is special power channel

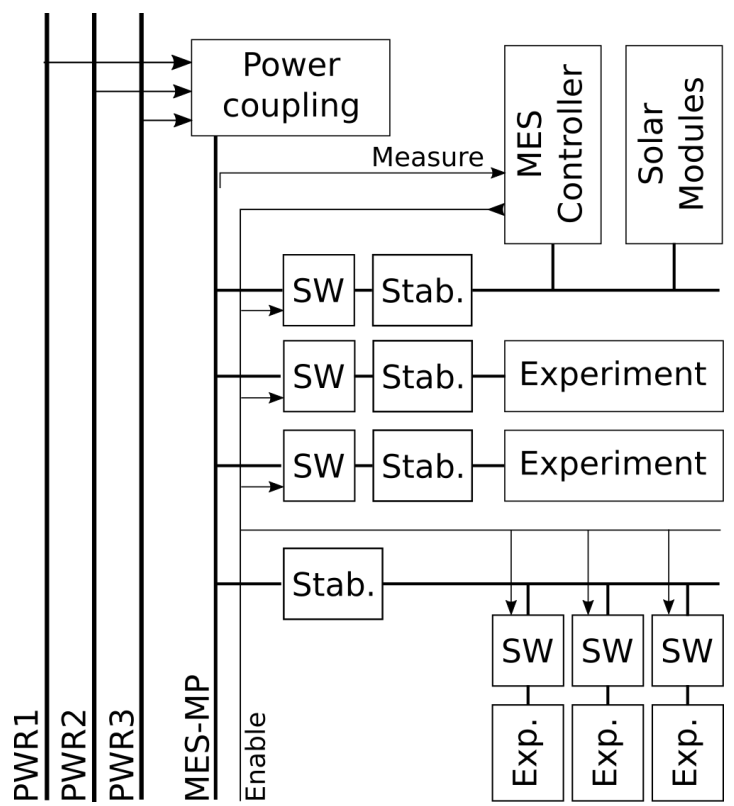

Fig. 6. Power system of the MES board 
for power supply of the MES Controller and Solar Modules that are basic parts of the MES board. There are two another types of power channels. The first type is used for experiments such as voltage references tests. It has its own switch followed by stabilizer. The second type of power channel is intended for more simple experiments such as Teploměrka. This power channel will supply more than one experiment from single stabilizer. In this case the stabilizer is followed by multiple switches (one switch for each experiment).

The coupling circuits are shown in more detail in the Fig.7. Construction of this part is based on LTC4411 integrated circuits, working as ideal diode. Coupled unstable voltage goes into measurement circuits. Measurement circuit perform voltage and current measurement signals and control signal $O N$ as well. $O N$ signa is main control signal that enable functions of all MES board subsystems. If there will be any power supply problem like under-voltage on the supply bus the $O N$ signal switch off whole MES board to protect another more crucial satellite subsystems like OBC or COM1. The $V+$ label is corresponding with MES Main Power bus in the Fig.6.

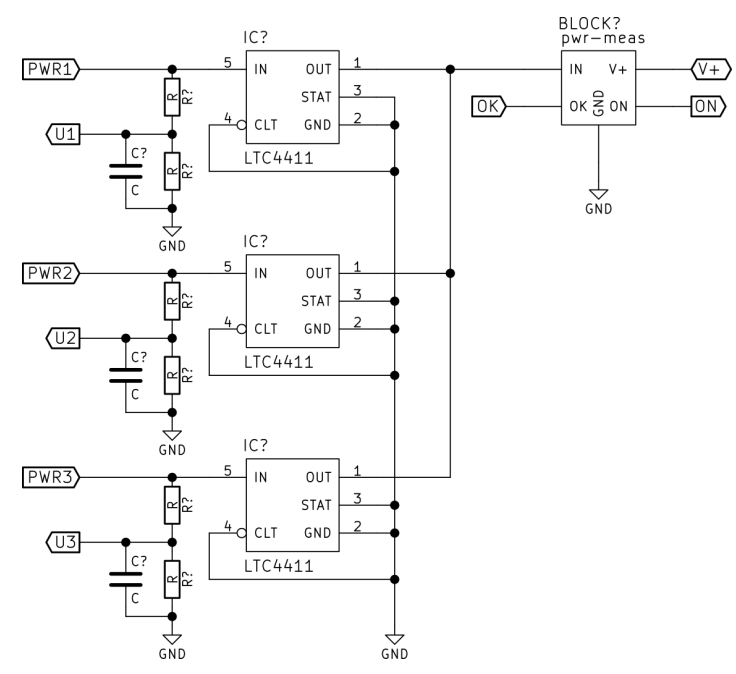

Fig. 7. PWR coupling circuits

The typical power channel for experiment is shown on the figures 8 and 9 . It is based on the integrated circuit STMPS2151 that work a power switch. Its function is enabled by signals $O N$ and $O K$. The signal $O N$ is generated in the power measurement and control block in the coupling circuits. Signal $O K$ is generated in the watchdog circuit that are checking function of main MES Controller. In case of MES Controller failure, the $O K$ signal switch off all experiments connected to it. After the SMTPS2151 power switch the power supply voltage is stabilized by MAX8877 integrated circuit. For more complex experiments the voltage labelled in the figure Fig.8 as VEXP is used as power supply.

In case of more simple experiments, the VEXP is used to supply more then one experiment. In such case there is one more SMTPS2151 power switch for every experiment. Typical configuration of these switches is shown in the Fig.9. These power switches are

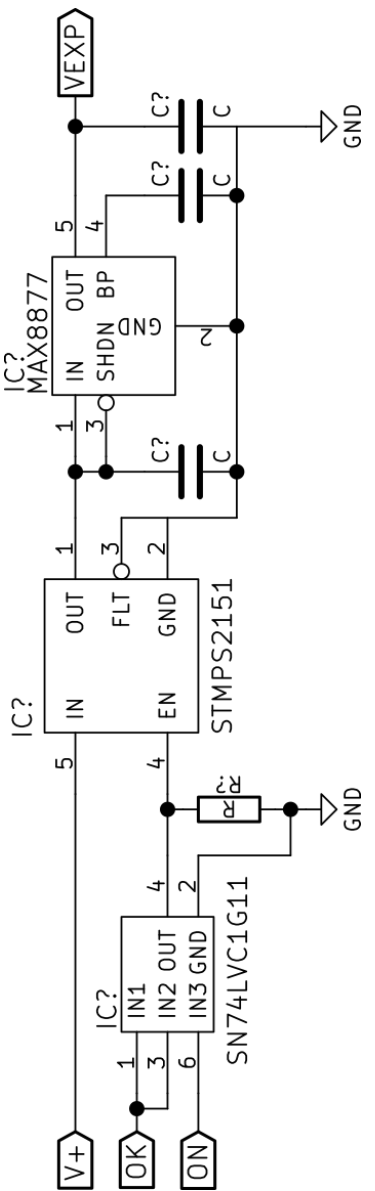

Fig. 8. Basic power supply channel.

controlled directly from MES Controller by the signals EXPEN. The EXPEN signal is generated independently for each experiment. The experiments connected to these switches are powered by the EXPP voltage.

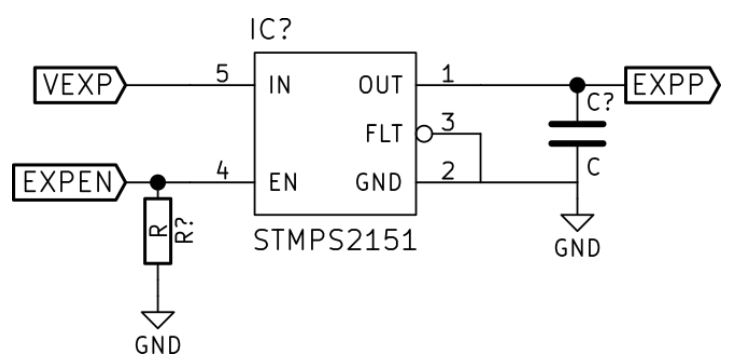

Fig. 9. Power channel switch.

\section{Solar Modules}

Solar modules are basic parts of the MES board. There will be installed 6 solar modules in the PilsenCUBE-II satellite. In each wall of the satellite will be one solar module containing various kinds of photosensitive components for sensing of radiation in infrared, visible and ultraviolet spectrum. These sensors will be used for attitude determination and earth position detection.

\section{E. Mechanical construction}

Mechanical construction of the MES board is derived from basic concept of the satellite. There is one 
main board with overall dimensions about 92 by 92 millimetres and a couple of additional boards for experiments testing microcontrollers (one for iSOUEsense and one for tested MSP430). Another additional boards are designed for solar modules which will be placed in each side of the satellite. These boards will contain various photodiodes and some STEs requiring free field of view into space such as experiment with Melexis temperature sensor. Last separated part will be $\mu C A M$ which is spatially demanding and require free view into space as well.

Mechanical construction of the board is quite challenging, because there will be a lot of sensors placed in very small place. In addition construction must be resistant to strong vibration during rocket launch. The printed circuit board will be carry out on the IS400 substrate in four layer configuration.

1) Main MES board: The mechanical dimensions and basic layout of main MES board is shown in the Fig.10. There are depicted basic mechanical parts for board embedding into satellite such as connectors for satelite bus, connectors for boards with tested microcontrollers and screw holes.

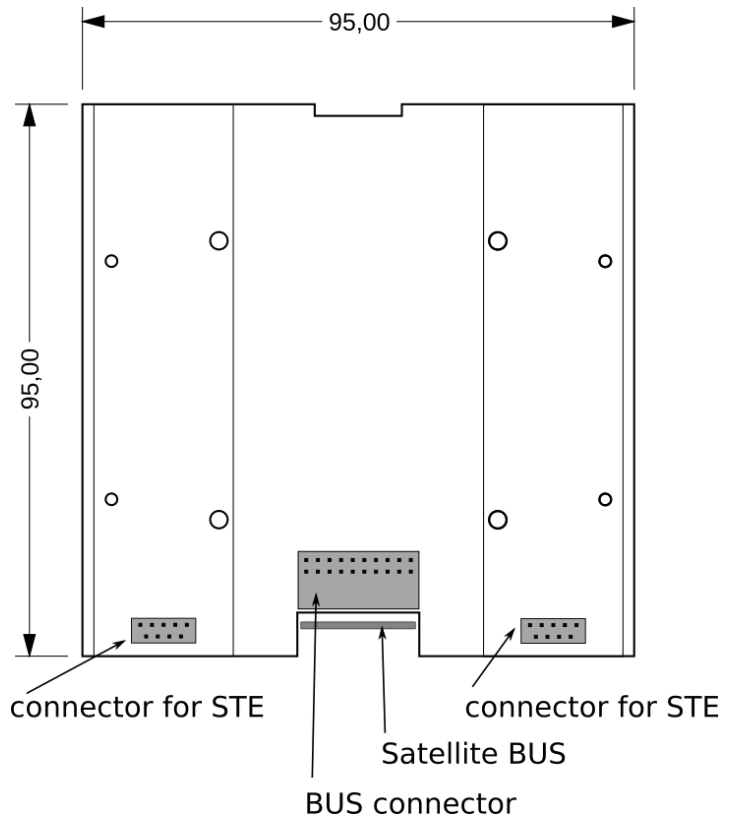

Fig. 10. MES board dimensions

The visualization if the Main MES board PCB is shown in the figure Fig.11.

2) Solar Modules: The construction of the solar modules is complicated by the different requirements for housing in the satellite walls. It is necessary to keep free field of view into space for solar modules sensors. Overall dimensions of the solar modules are not larger then 22 by 31 millimetres, but there can be some differences in design depending on module housing wall.

3) Boards for microcontroller testing: These two boards are designed by the teams preparing the microcontroller experiment. Still there are mechanical requirements enabling secure housing of these boards into satellite. Mechanical layout of these boards is

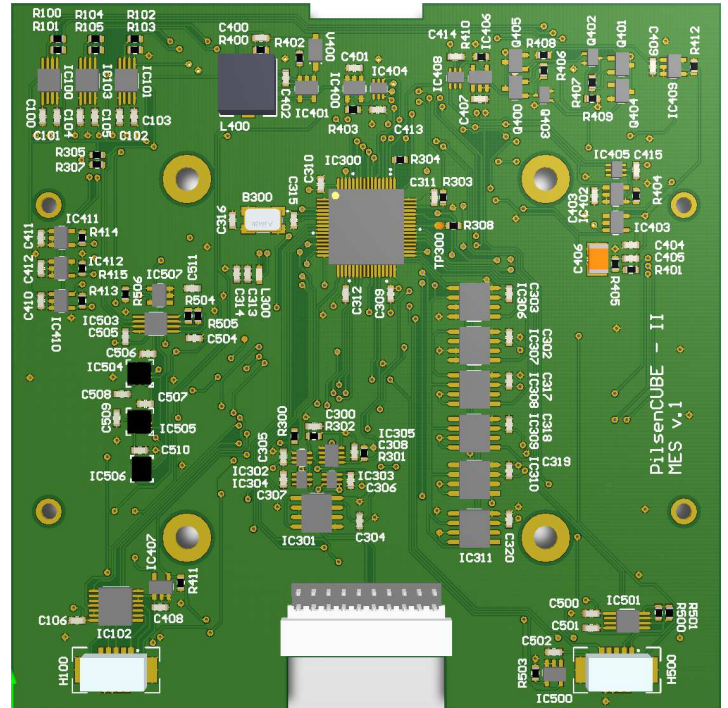

Fig. 11. Top side of the Main MES board PCB.

shown in the Fig.12. The overall dimensions of these boards are roundly 26 by 95 millimetres and its height must not exceed 4 millimetres.

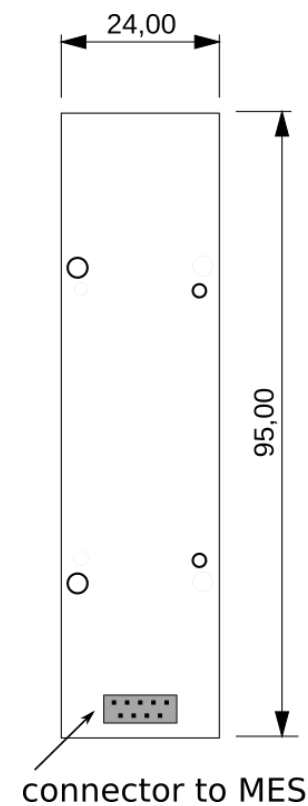

Fig. 12. Microcontroller testing boards dimensions

\section{F. Operation}

MES board function will be controlled from $\mathrm{OBC}$ by commands sended via satellite bus. These commands will turn on and of experiments separately according to scheduler, power consumption and overall satellite conditions.

Because there is large spectrum of the experiments controlled by the MES board and requirements of this experiments are very different it will be necessary perform control of each experiment individually with respect for its requirements.

There are experiments requiring relatively often, periodical measurements such as measurements from 
the solar modules, gyroscopes. Timing of these measurements will be adjusted according to speed of the satellite rotation which is expected in values of few rotations per minute. Another group of experiments can be activated continuously (if satellite conditions will be sufficient), but data from these experiments can be read out in relatively long periods such as tents of minutes. Into this group of experiments we can class experiments such as microcontroller testing, voltage reference testing and so on. The third group of experiments are event based experiments. Execution of these experiments is triggered by some event or sequence of events. For example taking of the photo by $\mu C A M$ will be enabled by OCB, but the camera triggering will wait until the camera will be pointing to the Earth.

\section{CONCLUSIONS}

PilsenCUBE-II Measurement board is complex payload part of satellite combining basic satellite measurement that is controlling and servicing connected experiments performed by various teams. It's main aim is carry out results of satellite attitude measurements and results from connected experiments. These results will be provided to $\mathrm{OBC}$ and COM1 boards that will report them to the ground station.

\section{ACKNOWLEDGEMENT}

This article was supported by project SGS-2018001 Research and development of the electronic and communication systems in science and engineering applications.

\section{REFERENCES}

[1] Ivo Veřtát, Richard Linhart, Luděk Dudáček, Vladimír Dániel, Petr Svoboda, "Autonomous and semi-autonomous radio commanding of VZLUSAT-1 nanosatellite from ground control station in Pilsen”, 2017 International Conference on Applied Electronics (AE). Pilsen 2017.

[2] Martin Matoušů, Ivo Veřtát, "Design of magnetic stabilization coils for PilsenCUBE picosatellite”, 2011 International Conference on Applied Electronics. Pilsen 2011.

[3] Ivo Veřtát, Jaroslav Hrouda, Jaroslav Hofman, "Spectrolab triangular solar cell evaluation for usage in PilsenCUBE picosatellite”, 2010 International Conference on Applied Electronics. Pilsen 2010.

[4] Pavel Fiala, Aleš Voborník, "Embedded microcontroller system for PilsenCUBE picosatellite”, 2013 IEEE 16th International Symposium on Design and Diagnostics of Electronic Circuits \& Systems (DDECS), 2013.

[5] Ivo Veřtát, Aleš Voborník, "Efficient and Reliable Solar Panels for Small CubeSat Picosatellites", Hindawi Publishing Corporation, International Journal of Photoenergy Volume 2014, Article ID 537645.

[6] Ivo Veřtát, Richard Linhart, Jiří Masopust, Aleš Voborník, Luděk Dudáček, "Earth's thermal radiation sensors for attitude determination system of small satellites", Contributions of the Astronomical Observatory Skalnate Pleso 47(2):157164, January 2017 